Aus der Chirurgischen Abteilung des Lehrkrankenhauses „Gülhane" in Konstantinopel.

\title{
Akuter lleus als erstes Krankheitssymptom bei Dünndarmtuberkulose und Bemerkungen über die Tuberkulose der Mesenterialdrüsen. ${ }^{1}$ )
}

Von Priv.-Doz. Stabsarzt Dr. Fritz Brüning, ehem. Chefarzt der Abteilung, z. Z. kommand. zur Chirurgischen Universitätsklinik der Charité in Berlin.

Jedem, der längere Zeit in der Türkei chirurgisch tätig war, muß die Häufigkeit der Lokalisation der Tuberkulose im Abdomen auffallen. Wenn ich sie auch nicht so häufig wie Wieting, mein Vorgänger am Krankenhaus Gülhane, beobachten konnte, so liegt dies daran, da $B$ seit Beginn meiner Tätigkeit im April 1915 das Gülhanekrankenhaus im wesentlichen als ein Militärlazarett arbeitete, also fast ausschließlich Soldaten behandelte, während es in Friedenszeiten ganz vorwiegend Zivilkranke aufnahm, unter denen naturgemä $\beta$ die Tuberkulose mehr verbreitet war als unter den jungen, kräftigen Soldaten.

Zunächst die Krankengeschichten in kurzem Auszug.

Im ersten Fall handelt es sich um einen 14 jährigen Kadetten, der vier Tage vor seiner Aufnahme mitErscheinungen von Darmokklusion erkrankt war; vorher hat er nie irgendwelche Störungen seiner Darmtätigkeit oder Schmerzen im Leibe gehabt.

Bei der Aufnahme zeigt sich eine starke Auftreibung des Leibes, Darmsteifungen deutlich sichtbar. Es besteht Erbrechen. Stuhl und Winde verhalten. Allgemeineindruck wie bei Peritonitis.

Sofort Operation: Es besteht eine frische, serös-fibrinöse Peritonitis. Im unteren Dünndarm eine Stenose, hervorgerufen durch eine stenosierende, zirkuläre Geschwürsbildung. Gleichzeitig an derselben Stelle starke Knickung des Darmes durch frische Verwachsungen. Hierdurch war die an sich nur relative Stenose in eine absolute umgewandelt worden. Resektion des erkrankten Darmteiles in Ausdehnung von etwa $30 \mathrm{~cm}$. Zirkuläre Darmnaht. Am aufgeschnittenen Präparat finden sich außer dem erwähntèn stenosierenden Geschwür noch unterhalb desselben zahlreiche oberflächliche Schleimhautgeschwüre, auch leider noch ganz in der Nähe des unteren Resektionsschnittes. Mikroskopisch wird später Tuberkulose nachgewiesen. Am zweiten Tag nach der Operation Abgang von Winden und reichlich Stuhl. Am vierten Tag plötzlich Verschlimmerung und Exitus an Peritonitis infolge Nahtinsuffizienz.

Im zweiten Fall handelt es sich um einen 25 jährigen Leutnant, der vor zwölf Stunden an sehr heftigen, plötzlich einsetzenden, kolikartigen Schmerzen erkrankt war. Auch er hat früher niemals irgendwelche Beschwerden in seiner Darmtätigkeit gehabt, er stand wegen beginnender Lungen: tuberkulose auf der Inneren Station in Behandlung. Da die kolikartigen Schmerzen andauerten und die Zeichen der Darmokklusion immer deutlicher wurden, interne Mittel, wie hohe Oeleinläufe usw., ohne Erfolg blieben, so wurde er 24 Stunden nach Beginn der Schmerzen operiert.

Operation: In der Bauchhöhle ein freier, seröser Erguß. Dünndarmschlingen stark gebläht, Dickdarm leer, kontrahiert. Im unteren Ileum findet sich eine Invagination, die sich nicht reponieren läßt. Resektion der erkrankten Partie in etwa $50 \mathrm{~cm}$ Ausdehnung, d. h. das ganze etwa: $20 \mathrm{~cm}$ lange Invaginatum und noch $30 \mathrm{~cm}$ Darm unterhalb desselben, soweit er entzündlich erkrankt aussieht und sich infiltriert anfühlt. Da nur noch wenige Zentimeter Dünndarm oberhalb der Bauhinischen Klappe stehen bleiben, so wird der Dünndarm seitlich in das Zökum implantiert. Reaktionslose Heilung.

Das Präparat zeigt nun die interessante Tatsache, da $\beta$ die Invagination durch ein stenosierendes, infiltrierendes, tuberkulöses Geschwür verursacht ist. Unterhalb der Invagination

1) Die Arbeit war schon im Herbst 1918 fast fertiggestellt. Die Drucklegung wurde verzögert durch meinen Fortgang von Konstantinoprl. 
zehlreiche Schleimhautgeschwüre, die denen im Fall 1 ganz ähnllch sind. Mikroskopisch werden sowohl in diesen flachen Schleimhautgeschwüren, wie auch in dem stenosierenden Geschwür auf der Höhe des Invaginatum typische Tuberkel mit Riesenzellen nachgewiesen. Das Invaginatum selbst zeigt schwerste Gewebsveränderungen im Sinne einer beginnenden Gangrän.

Was uns klinisch wohl am meisten interessiert, ist die Tatsache, daß in beiden Fällen eine tuberkulöse Darmerkrankung mit einem akuten Ileus eingesetzt hat, ohne daB vorher irgendwelche Krankheitssymptome bestanden hatten.

Daß es bei der Darmtuberkulose in vorgeschrittenen Stadien zum lleus kommen kann, ist ja allgemein bekannt. Meistens kommt dann allerdings ein sogenannter chronischer Ileus zustande. Bisweilen beobachtet man in solchen Fällen auch mal einen akuten Ileus. Er entwickelt sich dann aber meist aus dem chronischen, entweder infolge der zunehmenden Geschwürstenose oder infolge von Verwachsungen oder Strangbildung. Daß aber der akute Ileus das erste Krankheitszeichen einer Darmtuberkulose ist, stellt jedenfalls einen selten beobachteten Ausnahmefall dar. In dem bekannten von Wilms verfaßten Sammelwerk über Ileus habe ich eine solche Beobachtung nicht verzeichnet gefunden. Auch Wieting hat unter seinen Fällen nichts Derartiges gesehen.

In unserem ersten Fall war der akute Ileus dadurch bedingt daß sich zu der relativen Geschwürstenose eine durch frische Verwachsungen fixierte Darmknickung an der gleichen Stelle hinzugesellte

Im zweiten Fall, der von vornherein viel stürmischer verlief bedingte eine Invagination den Ileus. Die Entstehung der Invagination denke ich mir so: $D u r c h$ das zirkuläre infiltrierende Geschw ü war hier die Darmwand in ein starres Rohr verwandelt. Dieses starre Rohr wirkte nun wie ein Fremdkörper und wurde durch die oberhalb zur Ueberwindung der Stenose kräftiger arbeitende Darmtätigkeit wie ein Fremdkörper in den tiefern Darm$a b s c h n i t t h$ ineingedrängt. Auch diese Invagination im Dünndarm auf Grund einer Darmtuberkulose stellt ein sehr selten beobachtetes Ereignis dar. In jenem eben genannten Werk erwähnt Wilms nur zwei einschlägige Fälle aus der Literatur. An der Valvula ileocoecalis sind wohl solche Invaginationen öfters beobachtet worden, reine Dünndarm-Invagination auf Grund eines tuberkulösen Darmgeschwürs dürfen wir aber fraglos zu den Raritäten zählen.

In beiden Fällen war es ausgeschlossen, eine anatomische Diagnose zu stellen zumal beim Fehlen jeglicher anamnestischer Hinweise. An der Diagnose Darmverschluß war in beiden Fällen nicht zu zweifeln, und damit war die Indikation zur Operation gegeben.

Was den ungünstigen Ausgang im ersten Fall veranlaßt hat, läßt sich nicht mit Sicherheit sagen. Da schon bei der Operation eine Peritonitis bestand, ist es ja sehr wohl möglich, daß diese Peritonitis die Nahtinsuffizienz verschuldet hat. Es ist aber auch möglich, daß diese dadurch hervorgerufen ist, daß der untere Resektionsschnitt nicht im Gesunden lag und daß von den dort noch vorhandenen kleinen Schleimhautgeschwüren aus die Infektion der Nahtstelle und damit die Insuffizienz erfolgte. Bei der Operation glaubte ich bestimmt im Gesunden zu operieren. Weder durch die Inspektion der Darmwand von außen, noch durch Abtasten ließen sich Veränderungen an der Darmwand feststellen. Erst später am aufgeschnittenen Präparat sollte ich sehen, daß noch oberflächliche Schleimhautgeschwüre an der unteren Resektionsstelle saßen. Ich werde jedenfalls in Zukunft bei derartigen Prozessen recht radikal vorgehen.

Eine andere Frage ist nun die, ob man überhaupt resezieren oder sich lieber mit einer Umgehung der Stenose durch eine Enteroanastomose begnügen soll. Ich meine, wenn der Allgemeinzustand des Patienten es irgend zuläßt und wenn nicht durch weitergehende und anderweitige tuberkulöse Veränderungen eine Radikalheilung aussichtslos ist, so soll man immer resezieren. Denn sonst ist man - ganz abgesehen davon, daß man mit einer Enteroanastomose auf eine Radikalheilung verzichtet - nie davor sicher, daß nicht neue Komplikationen von seiten der Darmgeschwüre ein erneutes operatives Eingreifen erfordern, das dann natürlich viel weniger Aussicht auf Erfolg haben wird.

Bei einer Invagination ist die Entscheidung für unser operatives Vorgehen einfacher, denn es kommt dafür ja nur die Resektion in Frage.

Nun noch einiges über die reine MesenterialLymphdrüsentuberkulose, d. h. eine Lokalisation der Tuberkulose in diesen Drüsen, ohne daß sich sonst im Abdomen (z. B. am Peritoneum oder Darm usw.) tuberkulöse Veränderungen finden.

Wieting beschäftigt sích in seiner Festschrift ${ }^{1}$ ) auch mit

1) "Gülhane"-Festschrift zum 10 jährigen Bestehen des Kaiserl. Osmanischen Lehrkrankenhauses Gülhane. Leiprig 1909 bei Georg Thieme. dieser Erkrankung und veröffentlicht vier solcher Fulle, davon zwei (Fall 1 und 4), in denen nach einfacher Explorativ-Laparotomie mächtige Lymphknotenpakete völlig schwanden. In der Literatur hat Wieting keine derartigen Heilungen verzeichnet gefunden, nur einen Hinweis im Handbuch der speziellen Chirurgie von Koenig, wo es heiße: „Auch bei solchen Kranken (mit schwerer Erkrankung der Mesenteriallymphknoten, die einer Peritonealerkrankung zugrundelag), ist zuweilen nach Eröffnung des Bauches und Exstirpation einzelner Drüsen erhebliche Besserung eingetreten (Schmidt-Monard)."

Mir brachte die teilweise Exstirpation solcher tuberkulöser Drüsen in einem Fall auch eine ganz auffallende Besserung, von einer Heilung kann ich nicht reden, da der Kranke mehrere Monate nach der Operation einer sich plötzlich verschlimmernden, vorher symptomenlosen Lungentuberkulose erlag.

Leider ist das Krankenblatt bei meiner Ausreise aus der Türkei verloren gegangen, sodaß ich nur noch Folgendes aus meinem Gedächtnis über den Fall mitteilen kann.

Es handelt sich um einen 18 jährigen, kräftig entwickelten jungen Mann in recht gutem Ernährungszustand. Abgesehen von der gleich zu beschreibenden Geschwulst, waren an den inneren Organen, insbesondere an den Lungen, krankhafte Veränderungen nicht nachzuweisen. Rechts über und seitlich vom Nabel fühlte man einen gut faustgroßen, intraabdominal gelegenen, sich derb anfühlenden Tumor, der sich von Leber und Niere abgrenzen ließ und nur wenig verschieblich war. Man fühlte sehr kräftige Pulsation (fortgeleitet von der Aorta). Subjektiv bestanden ziehende Schmerzen von wechselnder Intensität. Keine Störungen der Darmtätigkeit. Es bestand unregelmäßiges Fieber

Bei der Operation (Laparotomie durch medianen Schnitt) zeigte sich das Peritoneum unverändert, auch waren am Darm keine Zeichen von Erkrankung zu finden. Der 'Tumor bestand aus einem Paket anscheinend tuberkulöser Lymphdrüsen. Da das Paket nach überallhin gut abgegrenzt erschien und auch keine Drüsenabszesse sich oberflächlich zeigten, so entschloß ich mich, die Exstirpation zu machen. Im weiteren Verlauf der Operation zeigte es sich jedoch, daB sich die erkrankten Drüsen sehr breit zwischen den beiden Blättern des Mesenteriums nach oben entwickelten. Da sich die Unterbindung mehrerer großer Darmgefäße nicht vermeiden ließ und mir bei weiterem Vorgehen die Ernährung des Darmes gefährdet schien, so begnügte ich mich mit einer teilweisen Exstirpation, sodaß ein etwa gänseeigroßer Teil zurückbleiben mußte. Nach sorgfältiger Blutstillung und Peritonisierung der Wunde wurde die Bauchhöhle durch Naht völlig geschlossen.

Die Wundheilung verlief ohne jede Störung. In wenigen Tagen nach der Operation ging die Temperatur zur Norm zurück. In den folgenden Wochen verkleinerte sich der zurückgebliebene Teil des Tumors, der sich zunächst noch sehr gut tasten ließ, so weit, daB nach drei Wochen auch nicht das Geringste mehr nachzuweisen war. Der Kranke war also als geheilt zu betrachten. Etwa zwei Monate nach der Operation zeigten sich die ersten Erscheinungen der Lungentuberkulose, welcher der Kranke sehr bald erlegen ist.

Dieser Fall bestätigt also ganz eklatant die Beobachtungen der weiter oben zitierten Autoren. Wir sehen an ihm, daß nach Laparotomie und teilweiser Exstirpation der erkrankten Drüsen der zurückgebliebene Rest später spontan geschwunden ist und daß die. Temperatur zur Norm zurückkehrte.

Ich meine, solche Beobachtungen lassen die Forderung berechtigt erscheinen, daß man bei der reinen Mesenterialdrüsentuberkulose eine Laparotomie - eventuell mit totaler oder partieller Exstirpation der Drüsen - ausführen soll, ganz wie es bei der Peritonitis tuberculosa fast allgemein geübt wird. Entschließt man sich zur Drüsenexstirpation, so $\mathrm{muB}$ man aber sorgfältig vermeiden, durch zu zahlreiche Gefäßunterbindungen die Ernährung der Darmwand $z u$ gefährden (vgl. Fall 2 bei Wieting). Bei drohender Gefahr muß man die Operation abbrechen und sich mit der partiellen Exstirpation begnügen, wozu man sich um so leichter entschließen kann, da. auch sie schon meist einen guten Erfolg verspricht.

Ich werde mich in Zukunft dann zur Exstirpation entschließen, wenn ich ein nicht zu großes, gut abgegrenztes Paket finde, dessen Exstirpation voraussichtlich ohne Schädigung der Darmwand möglich ist, und wenn diese Drüsenerkrankung anscheinend die einzige Lokalisation der Tuberkulose im Abdomen ist. in.allen anderen Fällen werde ich lediglich die Eröffnung der Bauchhöhle ausführen, wie das Wieting empfohlen hat.

Worin nun die Wirkung dieser Laparotomie besteht, das wissen wir ebensowenig wie für die Peritonitis tuberculosa. Mit Wieting können wir nur annehmen, daß dadurch eine Um- 
stímmung im Verhaltnis der Gewebe zu dem Infektionsherd zugunsten des ersteren geschaffen wird. 